\title{
Knockdown of sphingomyelin synthase 2 inhibits osteoclastogenesis by decreasing RANKL expression in mouse primary osteoblasts
}

\author{
Yoshihiro Yoshikawa ${ }^{1}$, Tatsuya Yoshizawa ${ }^{2}$, Eisuke Domae ${ }^{1}$, Yuya $\mathrm{Hirai}^{3}$, Aiko Kamada ${ }^{1}$, Toshiro Okazaki ${ }^{4}$, \\ and Takashi IKeO ${ }^{1}$ \\ ${ }^{1}$ Department of Biochemistry, Osaka Dental University, 8-1 Kuzuhahanazonocho, Hirakata 573-1121, Japan; ${ }^{2}$ Department of Medical \\ Biochemistry, Faculty of Life Sciences, Kumamoto University, Kumamoto 860-8556, Japan; ${ }^{3}$ Department of Biology, Osaka Dental \\ University, 8-1 Kuzuhahanazonocho, Hirakata 573-1121, Japan; and ${ }^{4}$ The Research Institute for Bioresources and Biotechnology, \\ Ishikawa Prefectural University, Ishikawa 921-8836, Japan
}

(Received 13 July 2019; and accepted 10 August 2019)

\begin{abstract}
Sphingomyelin is a major lipid of the plasma membrane and is enriched in microdomains of the plasma membrane that are critical for signal transduction. However, the function of sphingomyelin in the cell membrane of osteoblasts has not been clarified. Therefore, we examined how sphingomyelin synthase 2 (SMS2) affects osteoclast differentiation by osteoblasts. We knocked down the expression of SMS2 with siRNA targeting the Sgms 2 gene in mouse primary osteoblasts. The effects of SMS2 knockdown in osteoblasts were examined using polymerase chain reaction and western blotting. The knockdown of SMS2 suppressed the formation of TRAP-positive multinucleated cells by co-culture of osteoblasts and bone marrow cells compared to the control. We found that receptor activator of nuclear factor $\kappa B$ ligand (RANKL) mRNA expression was significantly reduced by $1,25(\mathrm{OH})_{2} \mathrm{D}_{3}$ stimulation in SMS2 siRNA osteoblasts. The knockdown of SMS2 repressed the expression of retinoid-X-receptor- $\alpha(\mathrm{RXR} \alpha)$ regardless of $1,25(\mathrm{OH})_{2} \mathrm{D}_{3}$ stimulation. TRAP-positive multinucleated cell formation was significantly reduced by $\mathrm{RXR} \alpha$ siRNA in osteoblasts in a co-culture system. These results suggest that SMS2 regulates osteoclast differentiation by inducing RANKL expression via RXR $\alpha$.
\end{abstract}

Bone is a dynamic tissue that undergoes continuous remodeling throughout the lifespan of humans. Bone mass is maintained through the balance between the functions of osteoblasts and osteoclasts. An imbalance between bone formation and resorption, therefore, results in various metabolic bone diseases (11). Bone metabolism is regulated by multiple hormones, growth factors, and cytokines.

Osteoclasts are multinucleated cells with a unique membrane known as the ruffled border membrane

Address correspondence to: Yoshihiro Yoshikawa, D.D.S., Ph.D., Department of Biochemistry, Osaka Dental University, 8-1 Kuzuhahanazonocho, Hirakata 573-1121, Japan

Tel: +81-72-864-3055, Fax: +81-72-864-3155

E-mail: y-yoshi@cc.osaka-dent.ac.jp with bone resorption function (26). They are formed by differentiation and fusion of monocyte-macrophage cells under the control of osteoblast-lineage cells (21). Differentiation from monocytes to osteoclasts is regulated by the receptor activator of nuclear factor $\mathrm{\kappa B}$ ligand (RANKL) and macrophage colony-stimulating factor (M-CSF). Bone resorption factors, including 1,25-dihydroxyvitamin D3 $\left(1,25(\mathrm{OH})_{2} \mathrm{D}_{3}\right)$, parathyroid hormone, interleukin (IL)-6, and IL-11, regulate the expression of RANKL and osteoprotegerin (OPG) genes in osteoblasts. In addition, they regulate calcium homeostasis, bone remodeling, proliferation, differentiation, and physiological processes $(10,19$, 28). $1,25(\mathrm{OH})_{2} \mathrm{D}_{3}$ binds to the vitamin $\mathrm{D}$ receptor (VDR) and promotes heterodimerization of VDR with a retinoid-X-receptor (RXR) to genomically regulate diverse cellular processes (9). 
Sphingolipids such as sphingomyelin, ceramide, and glycosphingosides are components of lipid bilayers. Sphingomyelin is the most abundant sphingolipid in the cell membrane and plays a role in maintaining its function and integrity. In recent years, sphingomyelin has been shown to be enriched in microdomains of cell membranes that are important for signal transduction, together with cholesterol (18). On the other hand, multiple stimuli cause the hydrolysis of sphingomyelin in the cell membrane to form ceramide, which is a second messenger (6). Thus, these sphingolipids participate in signaling associated with cell differentiation and proliferation (5). Sphingomyelin synthase (SMS) synthesizes sphingomyelin from ceramide, and of the two SMS (SMS1 and SMS2), SMS2 is present in the cell membrane $(7,29)$. SMS2 has been confirmed to be enriched in specific lipid microdomains, where it may regulate the function of these microdomains through sphingomyelin synthesis (27). Sphingomyelin turnover is induced by the activation of neutral sphingomyelinase (nSMase) during the induction of differentiation of the human leukemia HL-60 cell line by the addition of $1,25(\mathrm{OH})_{2} \mathrm{D}_{3}$ and the enhancement of ceramide production (16).

It remains unclear how SMS2 might be involved in osteoclast differentiation. Therefore, in this study, we investigated whether SMS2 affected osteoclast differentiation induced by $1,25(\mathrm{OH})_{2} \mathrm{D}_{3}$ in mouse primary osteoblasts.

\section{MATERIALS AND METHODS}

Animals. Slc:ddY mice were purchased from Shimizu Laboratory Supplies Co., Ltd. (Kyoto, Japan). Animal handling and experimental protocols were approved by the Animal Research Committee of Osaka Dental University.

Cell culture. Primary osteoblastic cells were isolated from the calvaria of 1-day-old male Slc:ddY mice and cultured in $\alpha$-MEM supplemented with $10 \%$ fetal bovine serum (FBS; Sigma-Aldrich, St. Louis, MO, USA), 100 unit $/ \mathrm{mL}$ penicillin $\mathrm{G}$ sodium, $100 \mu \mathrm{g} / \mathrm{mL}$ streptomycin, and $292 \mathrm{mg} / \mathrm{mL}$ L-glutamine (Invitrogen, Carlsbad, CA, USA) at $37^{\circ} \mathrm{C}$ in an atmosphere containing 5\% $\mathrm{CO}_{2}$. Bone-marrow cells obtained from the tibias of 6- to 8-week-old male Slc:ddY mice were cultured in $\alpha$-MEM containing 10\% FBS.

Antibodies and reagents. Anti-VDR (sc-13133), anti-RXR $\alpha$ (sc-553), and anti- $\beta$-actin antibodies (sc-
4778) were obtained from Santa Cruz Biotechnology (Paso Robles, CA, USA). Horseradish peroxidase (HRP)-labeled anti-rabbit secondary antibodies were obtained from Cell Signaling (Danvers, MA, USA). A Chemi-Lumi One Markers Kit was purchased from Nacalai Tesque (Kyoto, Japan).

Tartrate-resistant acid phosphatase (TRAP) buffer solution was prepared by mixing $0.1 \mathrm{M}$ sodium acetate (Wako Pure Chemicals, Osaka, Japan) and $50 \mathrm{mM}$ tartaric acid (Wako Pure Chemicals) in a $1: 1$ ratio, followed by adjustment with TRAP staining solution, which was prepared by mixing $50 \mathrm{~mL}$ of TRAP buffer solution, $5 \mathrm{mg}$ of naphthol AS-MX phosphate (Sigma-Aldrich), $0.5 \mathrm{~mL}$ of N,N-dimethylformamide (Wako Pure Chemicals), and $25 \mathrm{mg}$ of Fast Red Violet LB salt (Sigma-Aldrich).

Liquid chromatography tandem mass spectrometry (LC-MS/MS). Primary osteoblastic cells were homogenized in PBS. Protein was measured using a BCA protein assay kit (Pierce, Rockford, IL, USA), and cell lysate was processed for lipid extraction. Measurements of sphingomyelin species in lipid extracts were performed using LC-MS/MS as described previously $(14,15)$. The amounts of sphingomyelin species with various carbon chains (d18:1/16:0, d18:0/16:0, d18:1/18:1, d18:1/18:0, d18:1/20:1, d18:1/20:0, d18:1/22:1, d18:1/22:0, d18:1/24:1, d18:1/24:0, d18:1/26:1, d18:1/26:0, d16:1/18:1, $\mathrm{d} 16: 1 / 20: 0$, and $\mathrm{d} 18: 2 / 16: 0 \mathrm{~h})$ were determined. Each sphingomyelin was normalized to the total protein amount.

Osteoclast differentiation assay. Primary osteoblasts at a density of $2.5 \times 10^{3} /$ well and freshly prepared bone marrow cells at a density of $2.5 \times 10^{5} /$ well were cocultured in $\alpha$-MEM containing $10 \%$ FBS and $10^{-8} \mathrm{M} 1,25(\mathrm{OH})_{2} \mathrm{D}_{3}$ (Sigma-Aldrich) in 96-well plates. Osteoclasts formed within 7 days in cocultures. All cultures were incubated in quadruplicate, and medium was replenished on day 3. Cells were fixed in $4 \%$ paraformaldehyde in PBS (-) and an acetone/ethanol mixture $(1: 1)$. Fixed cells were treated with TRAP staining solution for $10 \mathrm{~min}$, washed with water, and observed by light microscopy. TRAP-positive multinucleated cells containing at least three nuclei were counted as osteoclasts. Representative results from at least three independent experiments are expressed as the mean \pm SEM of three cultures.

Western blotting. Cells were plated in 24-well tissue culture plates $\left(3.0 \times 10^{4} /\right.$ well $)$ and grown to subcon- 
fluency in $\alpha$-MEM supplemented with $10 \%$ FBS. Confluent monolayers were cultured in a medium containing $0.1 \%$ bovine serum albumin with $10^{-8} \mathrm{M}$ $1,25(\mathrm{OH})_{2} \mathrm{D}_{3}$ for $24 \mathrm{~h}$. Cells were washed twice with PBS and dissolved directly on the plate using sodium dodecyl sulfate (SDS) sample buffer and were briefly sonicated to shear DNA with a Branson digital sonifier 450 (Branson Ultrasonics, Danbury, CT, USA). Samples were boiled at $99^{\circ} \mathrm{C}$ for $3 \mathrm{~min}$ and proteins were separated by SDS-polyacrylamide gel electrophoresis. After transfer of the proteins to polyvinylidene difluoride membranes (Immobilon-P; Millipore, Texas City, TX, USA), membranes were blocked with 5\% skim milk in Tris-buffered saline (TBS) containing Tween-20 (TBS-T). Membranes were incubated overnight with primary antibody in $5 \%$ skim milk/TBS-T, followed by washing with TBS-T. Membranes were then incubated with peroxidase-conjugated secondary antibodies (Amersham Biosciences, Piscataway, NJ, USA) at a $1: 5000$ dilution for $1 \mathrm{~h}$, and immunoreactive bands were visualized using the Chemi-Lumi One Markers Kit (Nacalai Tesque). Signals were analyzed using a ChemiDoc XRS (Bio-Rad, Hercules, CA, USA). The same membranes were stripped and reprobed with antibodies recognizing $\beta$-actin for normalization of results.

Quantitative real-time reverse transcription (RT)$P C R$. Total RNA was isolated using an Illustra RNAspin Mini RNA Isolation Kit (GE Healthcare, Amersham, UK) from cultured cells at $24 \mathrm{~h}$ after the addition of $10^{-8} \mathrm{M} 1,25(\mathrm{OH})_{2} \mathrm{D}_{3}$. The quantity and purity of extracted total RNA were determined spectrophotometrically at 260 and $280 \mathrm{~nm}$. One microgram of total RNA was reverse-transcribed into cDNA using the RNA to cDNA EcoDry Premix (Clontech Laboratories, Inc., Palo Alto, CA, USA). Relative levels of mRNA expression were measured by quantitative real-time PCR using predesigned and preformulated gene-specific primer and probe sets for osteoclast differentiation factor molecules (TaqMan Gene Expression Assays; Applied Biosystems, Foster City, CA, USA). Analysis was performed with the StepOnePlus Real-Time PCR System (Applied Biosystems) in accordance with the manufacturer's protocol. Gene expression in multiplex reactions was quantified using the comparative $\mathrm{Ct}$ method with normalization of the amount of the target $\left(\mathrm{FAM}^{\mathrm{TM}}\right.$ ) to endogenous GAPDH (VIC ${ }^{\mathrm{TM}}$ ) expression. Probes for detecting cDNAs encoding RANKL, OPG, VDR, RXR $\alpha$, and GAPDH were designed and synthesized by Applied Biosystems, based on gene sequences in the Celera database. Assay IDs of TaqMan Gene Expression Assay probes were: RANKL, Mm00441908_m1; OPG, Mm00435452 m1; RXR $\alpha, M m 0044182^{-} \mathrm{m} 1$; VDR, Mm00437297 $\mathrm{m} 1$; and SMS2, Mm00512327_m1. The commercial number of TaqMan GAPDH control reagents was 4352932E.

Small interfering RNA (siRNA). Validated SMS2 (gene Sgms2) and RXRa (gene Rxra) siRNA and negative-control siRNA were purchased from Ambion (Grand Island, NY, USA) and transfected into cells using RNAi MAX reagent (Invitrogen) according to the manufacturer's protocol, without removal of medium for the duration of 1-day experiments. Knockdown of the target protein and gene was confirmed by immunoblotting and real-time PCR, respectively.

Statistical analysis. Results are presented as means \pm SDs of five experiments and were analyzed by unpaired Student's $t$-tests. Differences with $P$ values of less than 0.05 were considered significant.

\section{RESULTS}

SMS2 knockdown decreases sphingomyelin in primary osteoblasts

To determine whether regulation of osteoclast differentiation is dependent on SMS2, SMS2 expression was downregulated using Sgms2-targeted small interfering RNA (SMS2 siRNA) in primary osteoblasts. Successful knockdown was confirmed by RT-PCR (Fig. 1A). The levels of cellular sphingomyelin were measured by LC-MS/MS. As expected from the reduction of SMS2 expression, cellular sphingomyelin content was also reduced in SMS2 siRNA primary osteoblasts (Fig. 1B). These observations demonstrate that SMS2 and cellular sphingomyelin content was down-regulated by transient treatment with siRNA in primary osteoblasts.

SMS2 in primary osteoblasts potentiated osteoclast differentiation in cocultures with bone marrow cells Osteoclasts are generated when bone marrow cells and osteoblasts are cocultured in the presence of $1,25(\mathrm{OH})_{2} \mathrm{D}_{3}$. To determine whether osteoclast differentiation in response to a $1,25(\mathrm{OH})_{2} \mathrm{D}_{3}$ stimulus requires SMS2, we used an osteoclast coculture system in which primary osteoblasts were treated with siRNA negative control and SMS2 siRNA. SMS2 siRNA reduced the number of osteoclasts formed (Fig. 2). Taken together, these coculture data demon- 
A

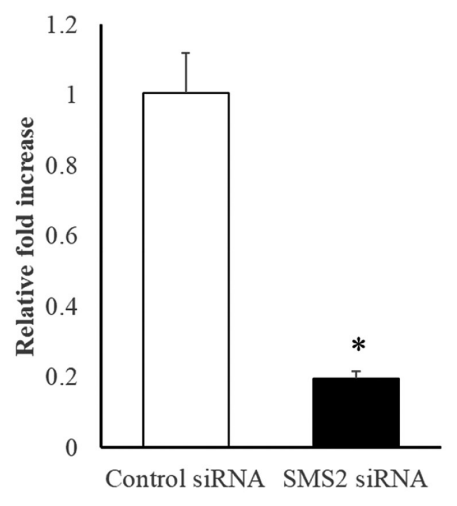

B

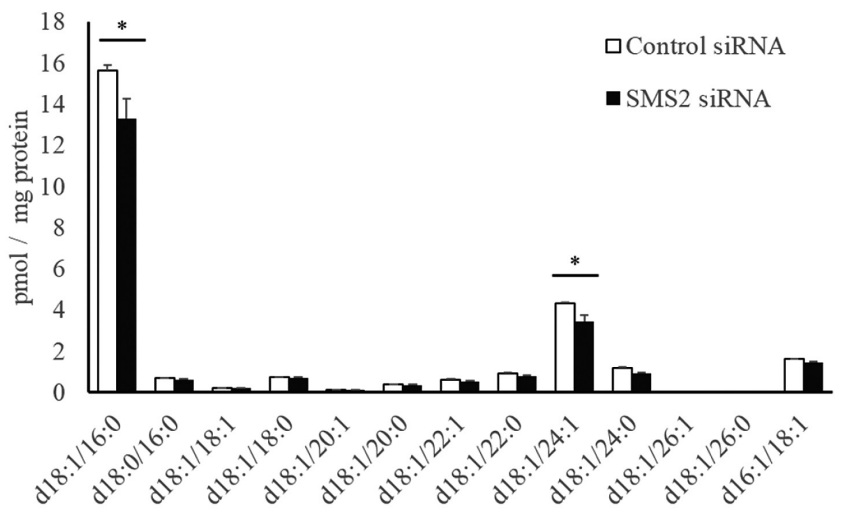

Fig. 1 Sphingomyelin expression is reduced by SMS2 siRNA in primary osteoblasts. Mouse calvarial osteoblasts were transfected with SMS2 siRNA or control siRNA for $24 \mathrm{~h}$. A. Gene expression of SMS2 mRNA was compared between control and SMS2 siRNA. B. The level of each molecular species of sphingomyelin was compared between control and SMS2 siRNA. Symbols represent mean values of four wells and vertical bars indicate SEMs. *Significant difference between the control and SMS2 siRNA groups $(P<0.05)$.

A

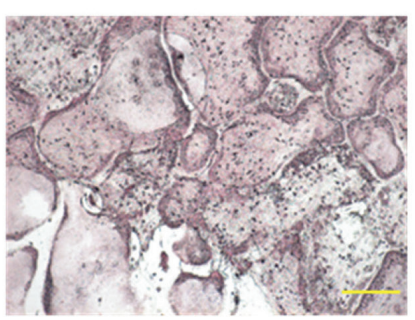

Control siRNA

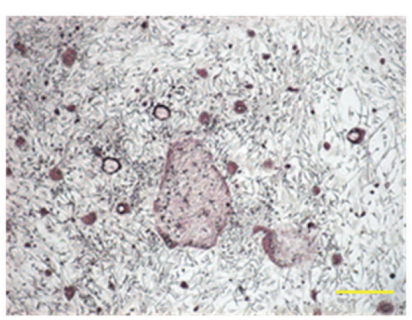

SMS2 SiRNA

B

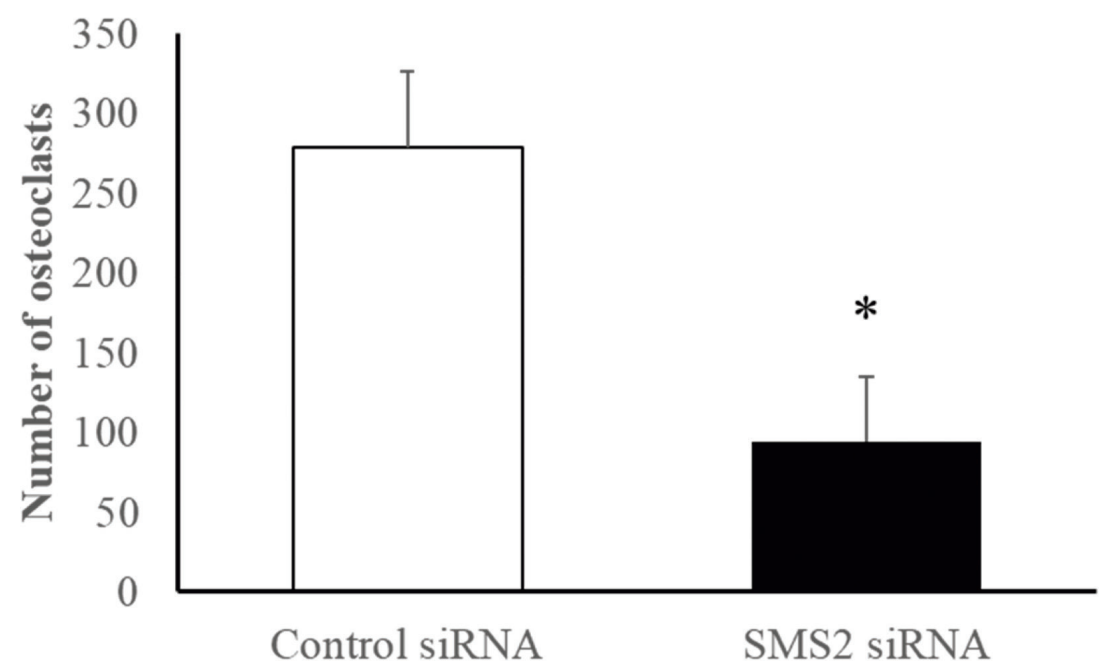

Fig. 2 SMS2 siRNA in primary osteoblasts blocked osteoclast formation. Mouse calvarial osteoblasts were transfected with SMS2 siRNA or control siRNA for $24 \mathrm{~h}$, and the osteoblast and bone marrow cell cocultures were then treated with $1,25(\mathrm{OH})_{2} \mathrm{D}_{3}$ for 7 days. A. Cells were fixed and stained for TRAP. B. The number of TRAP-positive multinucleated cells contain three or more nuclei were scored. Symbols represent mean values of four wells and vertical bars indicate SEM. *Significant difference between the control and SMS2 siRNA groups $(P<0.05)$. Scale bar $=250 \mu \mathrm{m}$. 
A

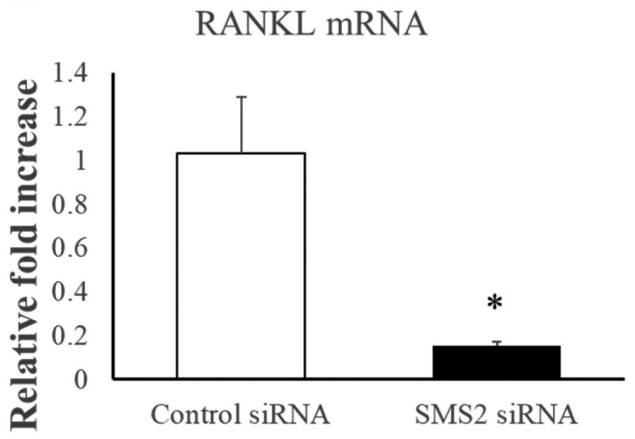

B

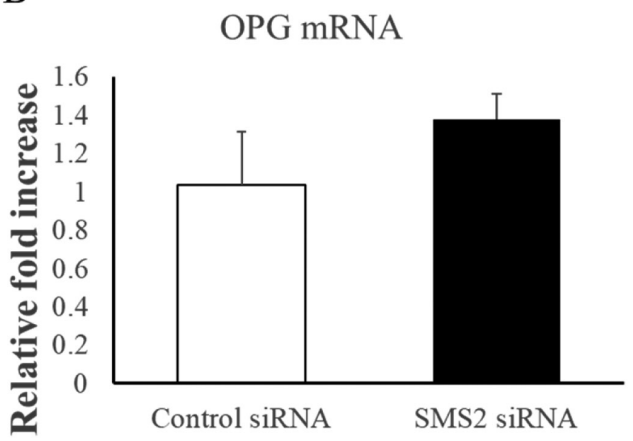

Fig. 3 SMS2 siRNA in primary osteoblasts blocked induction of RANKL gene expression in primary osteoblasts. Cells were transfected with SMS2 siRNA or control siRNA for $24 \mathrm{~h}$ and then treated with $1,25(\mathrm{OH})_{2} \mathrm{D}_{3}$ for $24 \mathrm{~h}$. RANKL and OPG mRNA expression was then detected by quantitative real-time PCR. Symbols represent mean values of four wells and vertical bars indicate SEMs. *Significant difference between the control and SMS2 siRNA groups $(P<0.05)$.

strate a functional role for SMS2 in osteoclast differentiation.

SMS2 knockdown inhibited RANKL mRNA expression in primary osteoblasts

To clarify the mechanisms involved in suppression of osteoclast differentiation by SMS2 siRNA, we investigated the effects of SMS2 siRNA on RANKL and OPG mRNA expression in primary osteoblasts. We observed a decrease in $1,25(\mathrm{OH})_{2} \mathrm{D}_{3}$ stimulated RANKL mRNA expression with SMS2 siRNA (Fig. 3A). Transfection with SMS2 siRNA did not change the induction of OPG mRNA expression (Fig. 3B). SMS2 siRNA affected RANKL, but not OPG, mRNA expression with $1,25(\mathrm{OH})_{2} \mathrm{D}_{3}$ in primary osteoblasts.

SMS2 siRNA inhibited RXR expression in primary osteoblasts

Although qPCR data showed that SMS2 is necessary for $1,25(\mathrm{OH})_{2} \mathrm{D}_{3}$ stimulated RANKL expression in osteoblasts, we sought to determine whether the requirement for SMS2 in $1,25(\mathrm{OH})_{2} \mathrm{D}_{3}$ signaling was limited to RANKL induction. We demonstrated that SMS2 siRNA in primary osteoblasts significantly decreased RXR $\alpha$ mRNA and protein expression (Fig. 4). However, neither VDR mRNA nor protein expression was changed (Fig. 4). These results indicate that SMS2 may be required for RXR $\alpha$ expression and regulate osteoclast differentiation.

$R X R \alpha$ in primary osteoblasts potentiated osteoclast differentiation in cocultures of bone marrow cells To determine whether primary osteoblasts are dependent on $\mathrm{RXR} \alpha$ for their ability to induce osteoclast differentiation in response to a $1,25(\mathrm{OH})_{2} \mathrm{D}_{3}$ stimulus, we used an osteoclast coculture system in which primary osteoblasts were treated with siRNA negative control and RXR $\alpha$ siRNA. RXR $\alpha$ siRNAtreated cocultures had a significant reduction in the number of osteoclasts formed (Fig. 5). Taken together, these coculture data demonstrate a functional role for RXR $\alpha$ in osteoclast differentiation.

\section{DISCUSSION}

In this study, we examined the effects of SMS2 on osteoclast differentiation induced by $1,25(\mathrm{OH})_{2} \mathrm{D}_{3}$ in mouse primary osteoblasts. Our results showed that SMS2 siRNA downregulated the expression of RXR $\alpha$, which heterodimerizes with VDR to influence RANKL mRNA expression in the presence of $1,25(\mathrm{OH})_{2} \mathrm{D}_{3}$. These effects ultimately resulted in enhanced osteoclast differentiation.

Previous studies have shown that SMS2 knockout mice causes type 2 diabetes by impairing glucose metabolism $(12,20)$. We have clarified that osteoblasts regulate glucose metabolism by ablation of osteoblasts that express osteocalcin (30), suggesting that SMS2 may influence osteoblastic function expression. SMS and nSMase contribute interconversion between ceramide and sphingomyelin (4). It supposed that activation of nSMase contributes increase of ceramide in this cycle, but it is pointed out significance of activation control of SMS recently (6, 24). In the present experiment, SMS2 siRNA led to a decrease in sphingomyelin levels in mouse primary osteoblasts.

Hydrolysis of sphingomyelin produces the intracellular signaling molecule ceramide. This triggers responses including cell death (13), differentiation (17), and inflammation (8). SMS2 is ubiquitous in 
A

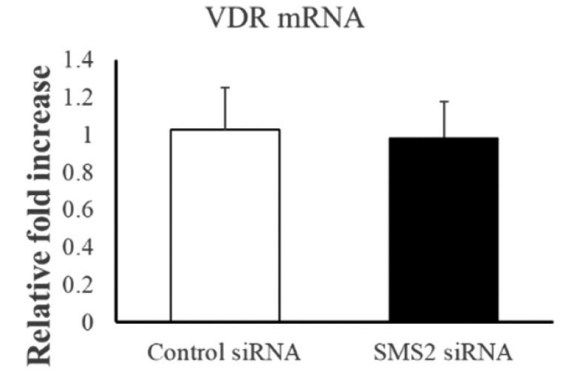

RXR $\alpha$ mRNA

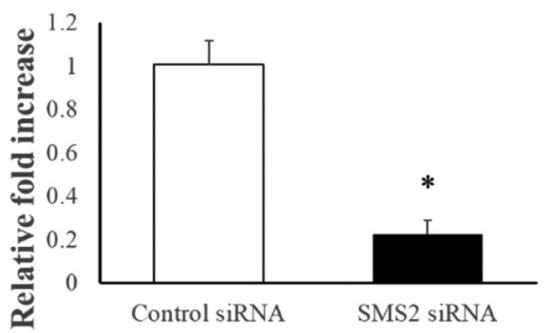

B

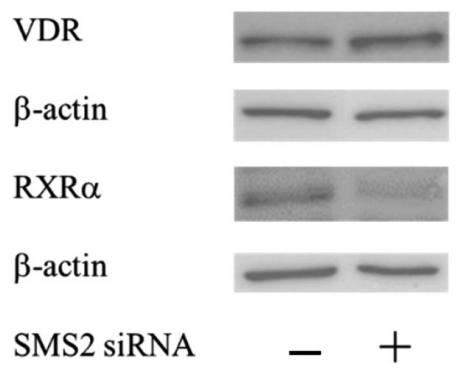

Fig. 4 SMS2 siRNA in primary osteoblasts inhibits RXRa expression. Cells were transfected with SMS2 siRNA or control siRNA for $24 \mathrm{~h}$ and then treated with $1,25(\mathrm{OH})_{2} \mathrm{D}_{3}$ for $24 \mathrm{~h}$. A. VDR and RXRa mRNA expression detected by quantitative real-time PCR. Symbols represent mean values of four wells, and vertical bars indicate SEMs. *Significant difference between the control and SMS2 siRNA groups $(P<0.05)$. B. Protein expression levels of VDR and RXRa were detected by western blot.

the plasma membrane and the Golgi apparatus, where it is responsible for sphingomyelin and diacylglycerol formation and ceramide consumption (29). This control of activation is important in the regulation of cell death and cell proliferation via these lipids. A previous report has suggested that such SMS2-dependent lipid regulation regulates inflammatory responses (3).

The human promyelocytic leukemia cell line HL60 has been widely used to study cell differentiation along the monocyte-macrophage pathway. $1,25(\mathrm{OH})_{2} \mathrm{D}_{3}$, tumor necrosis factor- $\alpha$ (TNF- $\alpha$ ), and $\gamma$-interferon induce differentiation of HL60 cells into monocytes $(1,23,25)$. Each of these differentiations is associated with changes in sphingolipid metabolism. Differentiation of HL60 cells into monocytes is regulated by a pathway involving sphingomyelin and its derivatives. In vitro, it has been confirmed that $1,25(\mathrm{OH})_{2} \mathrm{D}_{3}$ degrades sphingomyelin by activating $\mathrm{nSMase}$. In this case, ceramide functions as a second messenger (6). Furthermore, exogenous sphingomyelinase promoted differentiation to monocytes induced by $1,25(\mathrm{OH})_{2} \mathrm{D}_{3}$. Then we examined whether SMS2 affects osteoclast differentiation induced by $1,25(\mathrm{OH})_{2} \mathrm{D}_{3}$. We found that promotion of osteoclast differentiation was suppressed when SMS2 was knocked down in mouse primary osteoblasts. Thus, these data suggest that osteoclast differentiation is mediated by SMS2. Further analysis revealed that SMS2 knockdown inhibited the increase in RANKL mRNA expression caused by $1,25(\mathrm{OH})_{2} \mathrm{D}_{3}$ in mouse primary osteoblasts. These results indicated that induction of RANKL by $1,25(\mathrm{OH})_{2} \mathrm{D}_{3}$ was mediated by SMS2 signaling.

VDR bound to $1,25(\mathrm{OH})_{2} \mathrm{D}_{3}$ forms a heterodimer with the vitamin A receptor RXR $\alpha$, which then regulates transcription of target genes in the nuclei of osteoblasts (31). RXR $\alpha$ is a nuclear receptor that regulates the action of multiple endocrine processes through heterodimerization with a variety of hormone receptors, including thyroid receptor, peroxisome proliferator-activated receptor, VDR, and other retinoic-acid receptors (2). In addition, it has been reported that ceramide is involved in S1P phosphatase SPP in MC3T3-E1 cell line to control the transcriptional activity of AP-1 and increase retinoic acid receptor alpha and RXR $\alpha$ (22). Therefore, it is important to investigate whether SMS2 affects VDR and $\mathrm{RXR} \alpha$ protein expression. We observed no change in VDR protein expression with SMS2 knockdown, but $\mathrm{RXR} \alpha$ protein expression was decreased in mouse primary osteoblasts. RXR belongs to the nuclear receptor superfamily and has three isotypes: alpha, beta and gamma. Therefore, we examined the 
A

$\mathrm{B}$

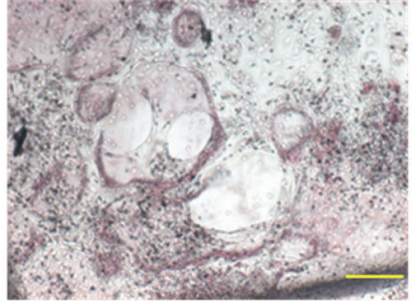

Control siRNA

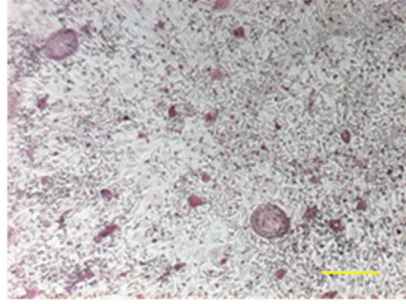

$\mathrm{RXR} \alpha$ SiRNA

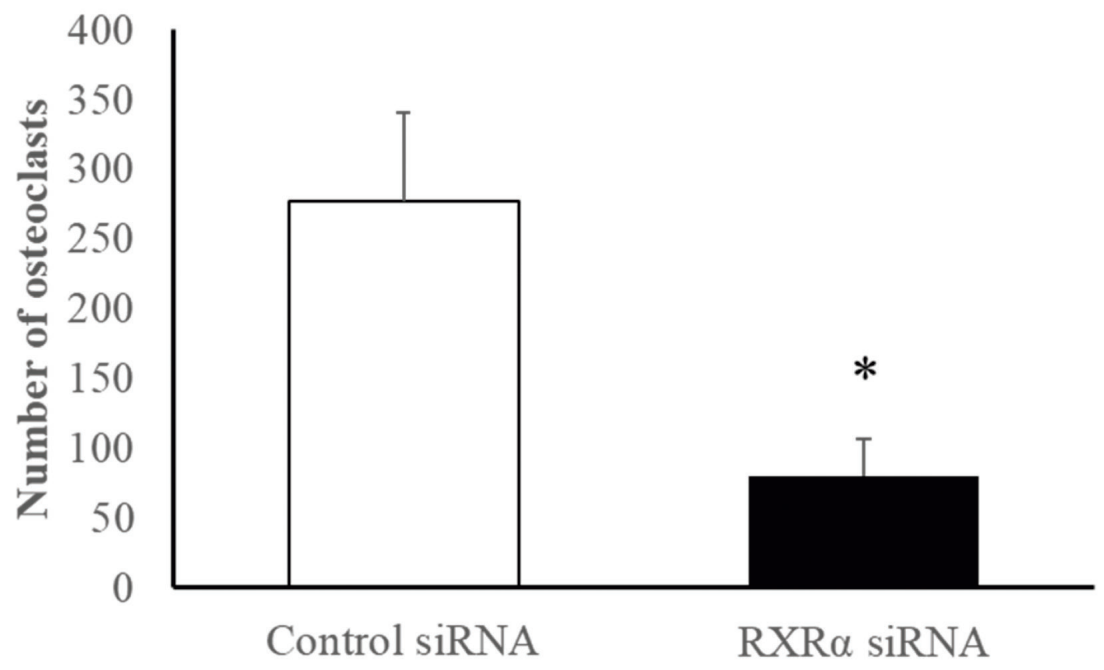

Fig. 5 RXRa siRNA in primary osteoblasts blocks osteoclast formation. Mouse calvarial osteoblasts were transfected with $\mathrm{RXRa}$ siRNA or control siRNA for $24 \mathrm{~h}$, and mouse calvarial osteoblast and bone marrow cell cocultures were then treated with $1,25(\mathrm{OH})_{2} \mathrm{D}_{3}$ for 7 days. A. Cells were fixed and stained for TRAP. B. The number of TRAP-positive multinucleated cells contain three or more nuclei were scored. Symbols represent mean values of four wells, and the vertical bars indicate SEM. *Significant difference between the control and RXRa siRNA groups $(P<0.05)$. Scale bar $=250 \mu \mathrm{m}$.

expression of all three, but no differences were found for the $\beta$ and $\gamma$ isotypes (data not shown). In summary, knockdown of SMS2 suppressed RXR $\alpha$ protein expression, but not VDR to which $1,25(\mathrm{OH})_{2} \mathrm{D}_{3}$ directly binds. Similarly, after SMS2 knockdown in mouse primary osteoblasts, RXR $\alpha$ mRNA expression was decreased. Therefore, when siRNA knockdown of RXR $\alpha$ was performed to confirm osteoclast differentiation, as expected, it was suppressed.

In conclusion, we have shown that knockdown of SMS2 reduces RXR $\alpha$ expression and decreases RANKL expression after $1,25(\mathrm{OH})_{2} \mathrm{D}_{3}$ stimulation in osteoblasts, which may suppress osteoclast differentiation. Further studies are needed to clarify the significance of SMS2 in the mechanism of RXR $\alpha$ expression regulation.

\section{Acknowledgements}

This study was partially supported by the Japan Society for the Promotion of Science, Grant-in-Aid for Scientific Research (C), grant number 17K12068.

\section{CONFLICTS OF INTEREST}

The authors declare that they have no conflicts of interest.

\section{REFERENCES}

1. Ball ED, Guyre PM, Shen L, Glynn JM, Maliszewski CR, Baker PE and Fanger MW (1984) Gamma interferon induces monocytoid differentiation in the HL-60 cell line. J Clin Invest 73, 1072-1077.

2. Evans RM (1988) The steroid and thyroid hormone receptor superfamily. Science 240, 889-895. 
3. Hailemariam TK, Huan C, Liu J, Li Z, Roman C, Kalbfeisch M, Bui HH, Peake DA, Kuo MS, Cao G, Wadgaonkar R and Jiang XC (2008) Sphingomyelin synthase 2 deficiency attenuates NFkappaB activation. Arterioscler Thromb Vasc Biol 28, 1519-1526.

4. Hannun YA (1994) The sphingomyelin cycle and the second messenger function of ceramide. $J$ Biol Chem 269, 31253128.

5. Hannun YA and Linardic CM (1993) Sphingolipid breakdown products: anti-proliferative and tumor-suppressor lipids. Biochim Biophys Acta 1154, 223-236.

6. Hannun YA and Obeid LM (2008) Principles of bioactive lipid signalling: lessons from sphingolipids. Nat Rev Mol Cell Biol 9, 139-150.

7. Huitema K, van den Dikkenberg J, Brouwers JF and Holthuis JC (2004) Identification of a family of animal sphingomyelin synthases. EMBO J 23, 33-44.

8. Kitatani K, Sheldon K, Anelli V, Jenkins RW, Sun Y, Grabowski GA, Obeid LM and Hannun YA (2009) Acid beta-glucosidase 1 counteracts p38delta-dependent induction of interleukin-6: possible role for ceramide as an anti-inflammatory lipid. J Biol Chem 284, 12979-12988.

9. Kliewer SA, Umesono K, Mangelsdorf DJ and Evans RM (1992) Retinoid X receptor interacts with nuclear receptors in retinoic acid, thyroid hormone and vitamin D3 signalling. Nature 355, 446-449.

10. Lacey DL, Timms E, Tan HL, Kelley MJ, Dunstan CR, Burgess T, Elliott R, Colombero A, Elliott G, Scully S, Hsu H, Sullivan J, Hawkins N, Davy E, Capparelli C, Eli A, Qian YX, Kaufman S, Sarosi I, Shalhoub V, Senaldi G, Guo J, Delaney J and Boyle WJ (1998) Osteoprotegerin ligand is a cytokine that regulates osteoclast differentiation and activation. Cell 93, 165-176.

11. Manolagas SC (2000) Birth and death of bone cells: basic regulatory mechanisms and implications for the pathogenesis and treatment of osteoporosis. Endocr Rev 21, 115-137.

12. Mitsutake S, Zama K, Yokota H, Yoshida T, Tanaka M, Mitsui M, Ikawa M, Okabe M, Tanaka Y, Yamashita T, Takemoto H, Okazaki T, Watanabe K and Igarashi Y (2011) Dynamic modification of sphingomyelin in lipid microdomains controls development of obesity, fatty liver, and type 2 diabetes. $J$ Biol Chem 286, 28544-28555.

13. Obeid LM, Linardic CM, Karolak LA and Hannun YA (1993) Programmed cell death induced by ceramide. Science $\mathbf{2 5 9}$, 1769-1771.

14. Ogiso H, Taniguchi M, Araya S, Aoki S, Wardhani LO, Yamashita Y, Ueda Y and Okazaki T (2014) Comparative analysis of biological sphingolipids with glycerophospholipids and diacylglycerol by LC-MS/MS. Metabolites 4, 98114.

15. Ogiso H, Taniguchi M and Okazaki T (2015) Analysis of lipidcomposition changes in plasma membrane microdomains. $J$ Lipid Res 56, 1594-1605.

16. Okazaki T, Bell RM and Hannun YA (1989) Sphingomyelin turnover induced by vitamin D3 in HL-60 cells. Role in cell differentiation. J Biol Chem 264, 19076-19080.

17. Okazaki T, Bielawska A, Bell RM and Hannun YA (1990) Role of ceramide as a lipid mediator of 1 alpha,25-dihydroxyvitamin D3-induced HL-60 cell differentiation. $J$ Biol Chem 265, 15823-15831.

18. Simons K and Gerl MJ (2010) Revitalizing membrane rafts: new tools and insights. Nat Rev Mol Cell Biol 11, 688-699.

19. Suda T, Takahashi N, Udagawa N, Jimi E, Gillespie MT and Martin TJ (1999) Modulation of osteoclast differentiation and function by the new members of the tumor necrosis factor receptor and ligand families. Endocr Rev 20, 345-357.

20. Sugimoto M, Shimizu Y, Zhao S, Ukon N, Nishijima K, Wakabayashi M, Yoshioka T, Higashino K, Numata Y, Okuda T, Tamaki N, Hanamatsu H, Igarashi Y and Kuge Y (2016) Characterization of the role of sphingomyelin synthase 2 in glucose metabolism in whole-body and peripheral tissues in mice. Biochim Biophys Acta 1861, 688-702.

21. Takahashi N, Akatsu T, Udagawa N, Sasaki T, Yamaguchi A, Moseley JM, Martin TJ and Suda T (1988) Osteoblastic cells are involved in osteoclast formation. Endocrinology 123, 2600-2602.

22. Takeshita A, Watanabe A, Takada Y and Hanazawa S (2000) Selective stimulation by ceramide of the expression of the alpha isoform of retinoic acid and retinoid $\mathrm{X}$ receptors in osteoblastic cells. A role of sphingosine 1-phosphate-mediated AP-1 in the ligand-dependent transcriptional activity of these receptors. J Biol Chem 275, 32220-32226.

23. Tanaka H, Abe E, Miyaura C, Shiina Y and Suda T (1983) 1 alpha,25-dihydroxyvitamin D3 induces differentiation of human promyelocytic leukemia cells (HL-60) into monocytemacrophages, but not into granulocytes. Biochem Biophys Res Commun 117, 86-92.

24. Taniguchi M and Okazaki T (2014) The role of sphingomyelin and sphingomyelin synthases in cell death, proliferation and migration-from cell and animal models to human disorders. Biochim Biophys Acta 1841, 692-703.

25. Trinchieri G, Kobayashi M, Rosen M, Loudon R, Murphy M and Perussia B (1986) Tumor necrosis factor and lymphotoxin induce differentiation of human myeloid cell lines in synergy with immune interferon. J Exp Med 164, 1206-1225.

26. Udagawa N, Takahashi N, Akatsu T, Tanaka H, Sasaki T, Nishihara T, Koga T, Martin TJ and Suda T (1990) Origin of osteoclasts: mature monocytes and macrophages are capable of differentiating into osteoclasts under a suitable microenvironment prepared by bone marrow-derived stromal cells. Proc Natl Acad Sci USA 87, 7260-7264.

27. Yamaoka S, Miyaji M, Kitano T, Umehara H and Okazaki T (2004) Expression cloning of a human cDNA restoring sphingomyelin synthesis and cell growth in sphingomyelin synthase-defective lymphoid cells. J Biol Chem 279, 1868818693.

28. Yasuda H, Shima N, Nakagawa N, Yamaguchi K, Kinosaki M, Mochizuki S, Tomoyasu A, Yano K, Goto M, Murakami A, Tsuda E, Morinaga T, Higashio K, Udagawa N, Takahashi N and Suda T (1998) Osteoclast differentiation factor is a ligand for osteoprotegerin/osteoclastogenesis-inhibitory factor and is identical to TRANCE/RANKL. Proc Natl Acad Sci USA 95, 3597-3602.

29. Yeang C, Varshney S, Wang R, Zhang Y, Ye D and Jiang XC (2008) The domain responsible for sphingomyelin synthase (SMS) activity. Biochim Biophys Acta 1781, 610-617.

30. Yoshikawa Y, Kode A, Xu L, Mosialou I, Silva BC, Ferron M, Clemens TL, Economides AN and Kousteni S (2011) Genetic evidence points to an osteocalcin-independent influence of osteoblasts on energy metabolism. J Bone Miner Res 26, 2012-2025.

31. Yoshikawa Y, Yoshizawa T, Domae E, Hieda Y, Takeyama A, Hirota S, Kawamoto A, Goda S, Tamura I, Kamada A, Komasa Y, Morita S, Yamagata K and Ikeo T (2015) RNA interference-mediated knockdown of Smad1 inhibits receptor activator of nuclear factor $\kappa \mathrm{B}$ ligand expression induced by BMP-2 in primary osteoblasts. Arch Oral Biol 60, 13191326. 